FACTA UNIVERSITATIS

Series: Physical Education and Sport, Vol. 16, No 2, 2018, pp. 245 - 260

https://doi.org/10.22190/FUPES180219022M

Research article

\title{
QUALITATIVE AND QUANTITATIVE EVALUATION OF THE CHARACTERISTICS OF THE ISOMETRIC MUSCLE FORCE OF DIFFERENT MUSCLE GROUPS IN CADET JUDO ATHLETES: A GENDER-BASED MULTIDIMENSIONAL MODEL
}

\author{
UDC 796.853.23:612.741
}

\section{Stefan Marković1, Milivoj Dopsaj ${ }^{1}{ }^{12}$, Vladimir Koprivica ${ }^{1}$, Goran Kasum ${ }^{1}$}

${ }^{1}$ Faculty of Sport and Physical Education, University of Belgrade, Belgrade, Serbia ${ }^{2}$ South Ural State University, Institute of Sport, Tourism and Service, Chelyabinsk, Russia

\begin{abstract}
The aim of this paper is to define the initial quantitative and qualitative multidimensional model for evaluating basic contractile characteristics of isometric muscle force in systematically trained and selected cadet judo athletes. In this research absolute values of the obtained results, and values derived by relativization of absolute values in relation to skeletal muscle mass (SMM) were considered. The basic method used in this research was laboratory testing. All data sampling was performed by the dynamometry method, using tensiometric probes. The research sample in this study consisted of 21 cadet judo athletes, of which 14 were male and 7 were female. All measurements were performed using standardized testing procedures on the following muscle groups: flexor muscles of the left (HGL) and the right hand (HGR), back (DL) and leg extensor muscles (LE) and ankle joint plantar flexor muscles (PF). Based on the obtained results, separate multidimensional mathematical models for the estimation of contractile potential and development level were defined for both basic characteristics of isometric muscle force: maximal isometric muscle force $\left(F_{\max }\right)$ and maximal explosive isometric muscle force $\left(R F D_{\max }\right)$. A qualitative assessment of contractile potential for each of the tested muscle groups, i.e. variables, was enabled by defining standard values for 7 distinct preparedness levels for both basic isometric muscle force contractile characteristics of male and female cadet judo athletes.
\end{abstract}

Key words: judo, cadets, isometric muscle force, standard values, model

Received February 19, 2018 / Accepted August 8, 2018

Corresponding author: Stefan Marković

University of Belgrade, Faculty of Sport and Physical Education, Blagoja Parovića 156, 11030 Belgrade, Serbia

Phone: +381 113531000 •E-mail: stephan.markovic@ hotmail.com 


\section{INTRODUCTION}

Judo is an Olympic martial sport that can be classified as a polystructural, acyclic sport discipline in which highly intensive intermittent work is dominant (Franchini, Del Vecchio, Matsushigue, \& Artioli, 2011). This is a dynamic, physically demanding sport, in which a high level performance requires complex motor skills and achieved tactical excellence (Cuk, Bratić, \& Mučibabić, 2016; Thomas, Cox, LeGal, Verde, \& Smith, 1989). From a physiological point of view, anaerobic power and capacity, as well as all the characteristics of muscle force and power, can be considered the main physical characteristics to be developed in judo competitors (Thomas et al., 1989) in order to achieve the necessary preconditions for achieving top results. A direct victory over an opponent in judo sport is primarily accomplished by the successful application of the throwing technique in a standing position, or by application of joint lock techniques or immobilizations in groundwork combat (Saraiva, Reis, Costa, Bentes, Costa e Silva, \& Novaes, 2014). Accordingly, the basic effort of the competitor during a judo contest is to throw the opponent on his back or control him on the ground (Franchini, Nunes, Moraes, \& Del Vecchio, 2007).

During a judo fight, the greatest number of explosive actions occur when an athlete attempts to defeat his/her opponent by rapid utilization of isolated or chained offensive actions or maneuvers (Monteiro, Chambel, \& Cardoso, 2011). The very use of the judo throwing technique depends on a number of preparatory elements, all aimed to achieving preconditions for unobstructed application of the throw. This can be accomplished through an effectively planed and implemented grip fighting (kumikata) which consumes about 50\% of the active fighting time (Calmet, Miarka, \& Franchini, 2010; Marcon, Franchini, Jardim, \& Barros Neto, 2010). Such an approach allows judo athletes to offbalance the opponent thus creating an opportunity for the execution of the throw which itself requires rapid actions (typically shorter than 1s) engaging upper and lower body muscle groups (Marcon et al., 2010; Blais, Trilles, \& Lacouture, 2007). In general, it can be said that for the efficient utilization of technique, it is necessary to apply it in the right manner, i.e. utilize it in the right moment, with a sufficient amount of strength and speed (Stamenković, Stanković, Nurkić, Nikolić, \& Petković, 2016).

Previous research indicates that approximately $70 \%$ of all injuries sustained in judo are a consequence of the throwing technique application (Pocecco et al., 2013) which further implies an irrefutable and crucial significance of this element in judo fighting, both in the context of sports achievement and in the context of sports preparation. Taking into account the complex nature of the judo sport (Milišić, 2003), as well as the fact that judo athletes overcome a significant external resistance in the form of actively resisting opponent, it is clear that achieving an adequate level of development of contractile characteristics of the involved musculature, both from the aspect of force (i.e. strength) and from the aspect of power, is one of the key elements that must be developed through a systematic training progression. This is in relation with the fact that muscular strength and power are considered the most highly ranked in the hierarchy of training abilities in successful judoka (Nurkić, Bratić, Mitić, Kafentarakis, 2017), and with the previous findings that indicate the utmost importance of muscle force and power training as a basic factor contributing to successful competitive performance both in judo and other martial sports (Detanico, Budal Arins, Dal Pupo, \& Dos Santos, 2012; Franchini, Del Vecchio, Matsushigue, \& Artioli, 2011; Laskowski \& Suchanowski, 2010; Thomas, Cox, LeGal, Verde, \& Smith, 1989). 
Based on the afore mentioned, a general conclusion can be drawn that the long-term preparation of an athlete in judo sport requires development of, at least, an adequate level of maximal muscle force of all the basic muscle groups. This can be considered a precondition for expressing a high level of the given contractile characteristic necessary for successful competition performance, as well as a foundation element contributing to injury prevention. However, recent research (Monteiro, 2016; Monteiro et al., 2011) indicates that a factor of key importance for achieving top results in judo can be the manifestation of a high level of development of explosive muscle force, i.e. explosiveness (RFD - rate of force development), which influences the effective execution of technique and the application of defensive maneuvers during the match.

According to the applicable rules of the International Judo Federation (IJF), competitors in judo are divided into 3 distinct age categories (cadets, juniors and seniors) in relation to chronological age (Sport and organization rules of the international judo federation, ed. 2017). This division is in compliance with the scientifically based concept of long-term sports career planning in which cadet age is considered initial in the system of sport selection and preparation (Koprivica, 2013; Dopsaj, 2015). Taking into account the afore mentioned and considering the fact that Umbrella sports organizations in judo sport, the European Judo Union (EJU) and the International Judo Federation (IJF), equally promote competitions for male and female competitors (Sindik, Rendulić, Čorak, \& Lewis, 2014), it is necessary to provide a system for the evaluation of basic contractile characteristics of muscle force at this initial age, as well as through the entire career progression, for both male and female competitors.

In accordance to the previous, this paper presents one of the initial studies aimed at defining the quantitative multidimensional model as well as the initial qualitative standard values in function of prediction and estimation of preparedness levels for both basic characteristics of isometric muscle force $\left(\mathrm{F}_{\max }\right.$ and $\left.\mathrm{RFD}_{\max }\right)$ in systematically trained and selected cadet judo athletes, both considering absolute values of the obtained results and values derived by relativization of absolute values in relation to skeletal muscle mass (SMM). Partialization of the results was enabled by the application of a new methodology for measuring the body structure of the participants using the InBody 720 system, which operates on the measurement principle of multichannel multisegmental bioelectric impedance (Dopsaj et al., 2015).

In this manner, relevant information is obtained, further increasing the body of scientific knowledge in this area and providing practical means for objective monitoring and improvement of the training process and long-term preparation in judo sport.

\section{METHODS}

The method used in this research was laboratory testing. All data sampling was performed by a dynamometry method, using tensiometric probes.

\section{The research sample}

The research sample in this study consisted of 21 cadet judo athletes, of which 14 were male and 7 were female. The basic descriptive characteristics for the male judo athlete subsample were: Age $=15.80 \pm 0.96$ years, $\mathrm{BH}=174.25 \pm 5.38 \mathrm{~cm}, \mathrm{BM}=66.71 \pm 6.01 \mathrm{~kg}, \mathrm{BMI}$ 
$=22.03 \pm 2.44 \mathrm{~kg} \cdot \mathrm{m}^{-2}, \mathrm{SMM}=35.48 \pm 3.57 \mathrm{~kg}, \mathrm{PSMM} \%=53.23 \pm 3.05 \%$, Training Experience $=4.06 \pm 2.24$ years, Weekly Training Frequency $=4.00 \pm 0.50$ sessions per week, Weekly Training Volume $=420.00 \pm 30.00$ minutes. The basic descriptive characteristics for the female judo athlete sub-sample were: Age $=16.90 \pm 0.57$ years, $\mathrm{BH}=159.87 \pm 7.79 \mathrm{~cm}$, $\mathrm{BM}=59.05 \pm 12.50 \mathrm{~kg}, \mathrm{BMI}=22.91 \pm 3.34 \mathrm{~kg} \cdot \mathrm{m}^{-2}, \mathrm{SMM}=26.37 \pm 3.84 \mathrm{~kg}, \mathrm{PSMM} \%=$ $45.38 \pm 5.45 \%$, Training Experience $=6.08 \pm 3.56$ years, Weekly Training Frequency $=4.00 \pm$ 0.50 sessions per week, Weekly Training Volume $=410.00 \pm 25.00$ minutes.

All of the participants were competing in the national championship system of the Republic of Serbia for the cadet age category. Two of the participants (one male and one female) were members of the Serbian cadet national team.

\section{Measurement protocols}

Before the beginning of this research, parents of all the participants (they were under the age of 18) read and signed an informed consent form. All of the participants and their coaches were informed in detail about the measurement procedures and the possible risks and benefits of this research. The study was approved by the Ethics Committee of the Faculty of Sport and Physical Education of the University of Belgrade. Testing of muscle force characteristics was performed using a dynamometry method, by application of isometric tensiometry, by means of tensiometric probes (Isometrics SMS All4Gym, Belgrade). All tests were performed at the University of Belgrade Faculty of Sport and Physical Education in the Methodological research laboratory (MIL). All tests were carried out in the morning hours by the application of a standardized testing procedure and standardized test battery described in previously published literature (Dopsaj, Milošević, Blagojević, 2000; Dopsaj et al., 2007; Marković, Dopsaj, \& Jovanović, 2017). For the purpose of avoiding negative effects of acute fatigue, all tested participants were not involved in any major physical activity 24 hours prior to the testing. Specific testing positions for each of the applied isometric muscle force tests are shown in pictures $1-4$.

Before the beginning of the testing, all of the participants performed $10 \mathrm{~min}$ of individual warm-up. After that the testing was carried out. The testing procedure required an explanation of the way each test is performed. Each participant had two trial attempts performed at high and submaximal intensity for the purpose of familiarization with the testing procedure. After having at least a $5 \mathrm{~min}$ rest, the testing was carried out using the trial-to-trial method, where the break between testing attempts was a minimum of $2 \mathrm{~min}$. Better test results were taken for statistical processing (Ivanović \& Dopsaj, 2013).

\section{Variables}

For the purposes of this paper, contractile characteristics of the isometric muscle force were examined from the aspect of the maximal level of its manifestation $\left(\mathrm{F}_{\max }\right)$ and from the aspect of the maximal level of intensity of its manifestation $\left(\mathrm{RFD}_{\max }\right)$. In other words, maximal isometric muscle force $\left(F_{\max }\right)$ and maximal explosive muscle force $\left(\mathrm{RFD}_{\max }\right)$ characteristics were examined. 


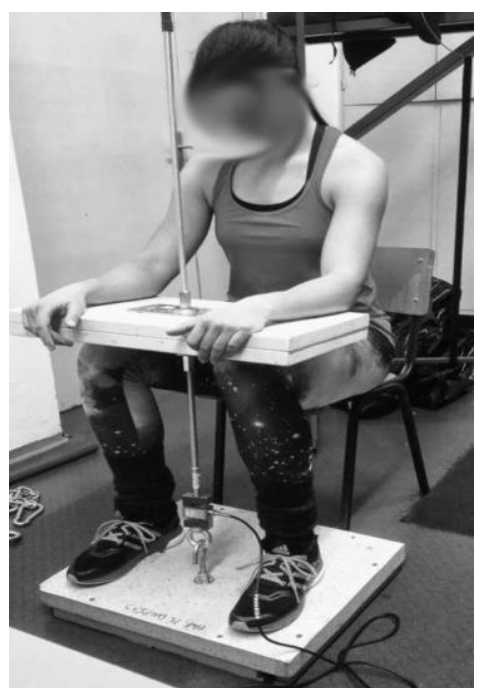

Fig. 1 Isometric Plantar Flexion, seated position

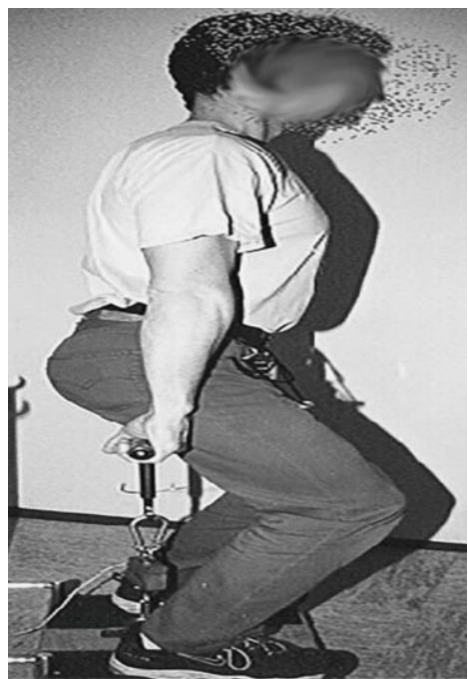

Fig. 3 Isometric Leg Extension, standing

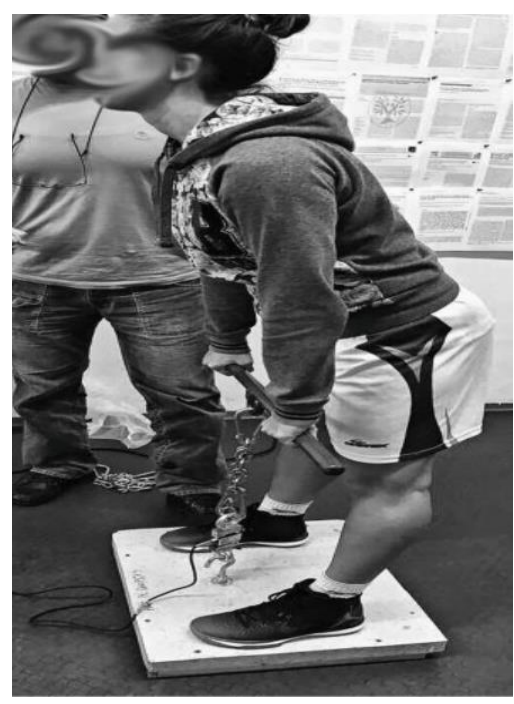

Fig. 2 Isometric Dead Lift

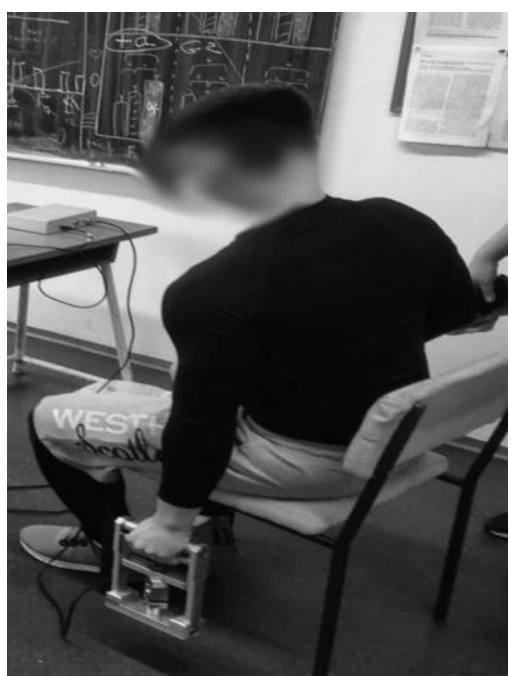

Fig. 4 Isometric Hand Grip, seated position

All tests were performed on 5 muscle groups as follows: flexor muscles of the left (HGL) and the right hand (HGR), back (DL) and leg (LE) extensor muscles and ankle joint plantar flexor muscles (PF).

The following variables were used for the measurement of maximal isometric muscle force:

- HGL_F $F_{\max }$ - maximal isometric force of the flexor muscles of the left hand expressed in $\mathrm{N}$;

- HGR_F $F_{\max }-$ maximal isometric force of the flexor muscles of the right hand expressed in $\mathrm{N}$; 
- DL_F $\mathrm{F}_{\max }$, maximal isometric force of back extensor muscles expressed in N;

- $L E \_F_{\max }$, maximal isometric force of leg extensor muscles expressed in $\mathrm{N}$;

- PF_F $\mathrm{F}_{\max }$, maximal isometric force of ankle joint plantar flexors expressed in $\mathrm{N}$;

The following variables were used for measurement of maximal explosive isometric muscle force (explosiveness):

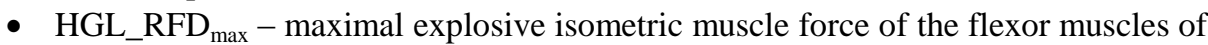
the left hand expressed in $\mathrm{N} \cdot \mathrm{s}^{-1}$;

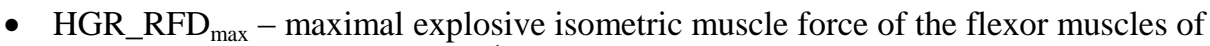
the right hand expressed in $\mathrm{N} \cdot \mathrm{s}^{-1}$;

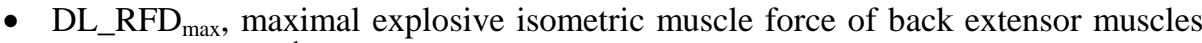
expressed in $\mathrm{N} \cdot \mathrm{s}^{-1}$;

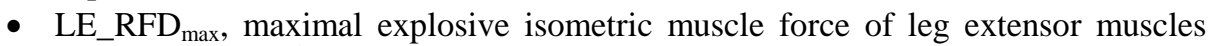
expressed in $\mathrm{N} \cdot \mathrm{s}^{-1}$;

- PF_RFD $D_{\max }$, maximal explosive isometric muscle force of ankle joint plantar flexors expressed in $\mathrm{N} \cdot \mathrm{s}^{-1}$;

All absolute values of the examined variables were partialized relative to pure skeletal muscle mass (SMM) in order to obtain relevant data in relation to the used component of the body which is directly responsible for the manifestation of the contractile potential of skeletal muscles. All calculations were done using the standard calculation formula as follows: VAR_CC $C_{\text {rel }}=$ VAR_CC $C_{\max } / \mathrm{SMM}$, where VAR_CC $\mathrm{Cax}_{\max }$ represents the measured value of the isometric muscle force characteristic for the given muscle group, SMM represents skeletal muscle mass expressed in $\mathrm{kg}$, while VAR_ $\mathrm{CC}_{\text {rel }}$ represents the value of the variable derived.

\section{Statistical analysis}

For the purposes of this paper, in the first step of the analysis all raw data obtained by laboratory testing were subjected to descriptive statistical analysis in order to define the basic measure of central tendency (MEAN), indicators of data dispersion - standard deviation and coefficient of variation $(\mathrm{SD}, \mathrm{cV} \%)$ and result span indicators - minimum and maximum (MIN, MAX). Normality of the distribution of the results was estimated for each variable by application of the Shapiro-Wilk test. Based on the obtained results, for each of the tested muscle groups, i.e. variables, the initial qualitative standard values were defined using adequate sport metrological procedures (Зациорски, 1982). In the next step of analysis, mathematical modeling by means of multidimensional scaling was used to define the total numerical score of general fitness in function of each measured contractile characteristic (Dopsaj et al., 2010; Dopsaj, 2015). In this way general preparedness for both maximal isometric muscle force (Bod_SCORE_F $F_{\max }$ ) and maximal isometric explosive muscle force (Bod_SCORE_RFD ${ }_{\max }$ ) was presented by mathematical analogy where the value of test score for each individual participant was transformed into a proportional point score on a scale from 0 (hypothetical minimum) to 100 (hypothetical maximum) points. The final form of the mathematical model was defined by application of the Multivariate Regression Analysis (MRA), where the value of point score represented the criterion variable, and the results of 5 isometric tests represented a system of predictor variables. All analysis were conducted by use of statistical packages Microsoft Office Excel 2013 and IBM SPSS v23.0. 


\section{RESULTS}

The results of descriptive statistical analysis relative to the examined absolute and relative variables of maximal isometric muscle force $\left(\mathrm{F}_{\max }\right)$ with the results of the Shapiro-Wilk goodness of fit test, for both male and female judo athletes, are shown in Table 1.

Table 1 Descriptive statistics for the maximal isometric muscle force $\left(F_{\max }\right)$ variables with the results of the Shapiro-Wilk normality test

\begin{tabular}{|c|c|c|c|c|c|c|c|c|}
\hline \multicolumn{9}{|c|}{ Male Judo Athletes } \\
\hline \multicolumn{9}{|c|}{ Absolute values } \\
\hline & $\mathrm{N}$ & MEAN & SD & $\% \mathrm{cV}$ & MIN & MAX & S-W & $\mathrm{p}$ \\
\hline$\overline{\mathrm{HGL}} \mathrm{F}_{\max }(\mathrm{N})$ & 14 & 427.23 & 97.04 & 22.71 & 281.00 & 558.00 & 0.905 & 0.133 \\
\hline $\mathrm{HGR}_{-} \mathrm{F}_{\max }(\mathrm{N})$ & 14 & 448.92 & 100.58 & 22.40 & 305.00 & 641.00 & 0.965 & 0.804 \\
\hline $\mathrm{DL} \_\mathrm{F}_{\max }(\mathrm{N})$ & 14 & 1264.62 & 299.25 & 23.66 & 845.00 & 1779.00 & 0.952 & 0.594 \\
\hline $\mathrm{LE} \_\mathrm{F}_{\max }(\mathrm{N})$ & 14 & 1151.54 & 254.22 & 22.08 & 723.00 & 1628.00 & 0.990 & 1.000 \\
\hline PF_F $F_{\max }(N)$ & 14 & 3177.54 & 786.16 & 24.74 & 2228.00 & 4957.00 & 0.877 & 0.053 \\
\hline \multicolumn{9}{|c|}{ Relative values } \\
\hline HGL_F $\mathrm{F}_{\text {rel }}\left(\mathrm{N} \cdot \mathrm{kg}_{\mathrm{SMM}}{ }^{-1}\right)$ & 14 & 12.00 & 2.29 & 19.06 & 8.17 & 15.87 & 0.965 & 0.806 \\
\hline HGR_F $F_{\text {rel }}\left(\mathrm{N} \cdot \mathrm{kg}_{\mathrm{SMM}}{ }^{-1}\right)$ & 14 & 12.57 & 2.10 & 16.72 & 9.76 & 16.52 & 0.939 & 0.405 \\
\hline $\mathrm{DL} \_\mathrm{F}_{\mathrm{rel}}\left(\mathrm{N} \cdot \mathrm{kg}_{\mathrm{SMM}}{ }^{-1}\right)$ & 14 & 35.42 & 6.52 & 18.39 & 24.56 & 47.06 & 0.970 & 0.881 \\
\hline LE_F $F_{\text {rel }}\left(\mathrm{N} \cdot \mathrm{kg}_{\mathrm{SMM}}{ }^{-1}\right)$ & 14 & 32.25 & 5.20 & 16.12 & 22.88 & 43.07 & 0.924 & 0.249 \\
\hline PF_F $F_{\text {rel }}\left(\mathrm{N} \cdot \mathrm{kg}_{\mathrm{SMM}}{ }^{-1}\right)$ & 14 & 89.58 & 20.70 & 23.10 & 72.45 & 134.70 & 0.768 & 0.002 \\
\hline \multicolumn{9}{|c|}{ Female Judo Athletes } \\
\hline \multicolumn{9}{|c|}{ Absolute values } \\
\hline & $\mathrm{N}$ & MEAN & SD & $\% \mathrm{cV}$ & MIN & MAX & S-W & $\mathrm{p}$ \\
\hline $\mathrm{HGL}_{\mathrm{H}} \mathrm{F}_{\max }(\mathrm{N})$ & 7 & 240.83 & 64.70 & 26.86 & 182.00 & 348.00 & 0.852 & 0.128 \\
\hline $\mathrm{HGR}_{-} \mathrm{F}_{\max }(\mathrm{N})$ & 7 & 241.83 & 47.61 & 19.69 & 195.00 & 333.00 & 0.889 & 0.272 \\
\hline $\mathrm{DL} \_\mathrm{F}_{\max }(\mathrm{N})$ & 7 & 714.50 & 76.04 & 10.64 & 612.00 & 844.00 & 0.965 & 0.859 \\
\hline LE_F $F_{\max }(\mathrm{N})$ & 7 & 634.00 & 99.29 & 15.66 & 530.00 & 779.00 & 0.887 & 0.259 \\
\hline PF_F $F_{\max }(N)$ & 7 & 2917.17 & 572.43 & 19.62 & 2435.00 & 4000.00 & 0.844 & 0.107 \\
\hline \multicolumn{9}{|c|}{ Relative values } \\
\hline HGL_F $F_{\text {rel }}\left(\mathrm{N} \cdot \mathrm{kg}_{\text {SMM }^{-1}}\right)$ & 7 & 9.22 & 2.33 & 25.26 & 6.04 & 12.30 & 0.954 & 0.764 \\
\hline HGR_F $F_{\text {rel }}\left(\mathrm{N} \cdot \mathrm{kg}_{\mathrm{SMM}}{ }^{-1}\right)$ & 7 & 9.35 & 2.21 & 23.70 & 6.19 & 12.51 & 0.956 & 0.788 \\
\hline $\mathrm{DL} \_\mathrm{F}_{\text {rel }}\left(\mathrm{N} \cdot \mathrm{kg}_{\mathrm{SMM}}{ }^{-1}\right)$ & 7 & 27.41 & 3.41 & 12.43 & 21.80 & 32.26 & 0.982 & 0.969 \\
\hline LE_F $F_{\text {rel }}\left(\mathrm{N} \cdot \mathrm{kg}_{\mathrm{SMM}}{ }^{-1}\right)$ & 7 & 24.47 & 4.82 & 19.71 & 16.56 & 31.11 & 0.972 & 0.912 \\
\hline PF_F $F_{\text {rel }}\left(\mathrm{N} \cdot \mathrm{kg}_{\mathrm{SMM}}{ }^{-1}\right)$ & 7 & 111.71 & 20.86 & 18.68 & 82.51 & 143.88 & 0.991 & 0.995 \\
\hline
\end{tabular}


The results of the descriptive statistical analysis relative to the examined absolute and relative variables of maximal explosive isometric muscle force $\left(\mathrm{RFD}_{\max }\right)$, with the results of the Shapiro-Wilk goodness of fit test, for both male and female judo athletes, are shown in Table 2.

Table 2 Descriptive statistics for the maximal isometric explosive muscle force $\left(\mathrm{RFD}_{\max }\right)$ variables with the results of the Shapiro-Wilk normality test

\begin{tabular}{|c|c|c|c|c|c|c|c|c|}
\hline \multicolumn{9}{|c|}{ Male Judo Athletes } \\
\hline \multicolumn{9}{|c|}{ Absolute values } \\
\hline & $\mathrm{N}$ & MEAN & SD & $\% \mathrm{cV}$ & MIN & MAX & S-W & $\mathrm{p}$ \\
\hline HGL_RFD ${ }_{\max }\left(\mathrm{N} \cdot \mathrm{s}^{-1}\right)$ & 14 & 2614.85 & 740.27 & 28.31 & 1776 & 3748 & 0.881 & 0.060 \\
\hline HGR_RFD ${ }_{\max }\left(\mathrm{N} \cdot \mathrm{s}^{-1}\right)$ & 14 & 2658.85 & 795.60 & 29.92 & 1779 & 4261 & 0.900 & 0.112 \\
\hline $\mathrm{DL} \_\mathrm{RFD}_{\max }\left(\mathrm{N} \cdot \mathrm{s}^{-1}\right)$ & 14 & 6697.69 & 2810.77 & 41.97 & 3673 & 13679 & 0.878 & 0.054 \\
\hline LE_RFD ${ }_{\max }\left(\mathrm{N} \cdot \mathrm{s}^{-1}\right)$ & 14 & 4852.23 & 1238.52 & 25.52 & 3329 & 7425 & 0.920 & 0.221 \\
\hline PF_RFD ${ }_{\max }\left(\mathrm{N} \cdot \mathrm{s}^{-1}\right)$ & 14 & 14252.08 & 3634.04 & 25.50 & 9819 & 22557 & 0.918 & 0.205 \\
\hline \multicolumn{9}{|c|}{$\begin{array}{l}\text { Relative values } \\
\end{array}$} \\
\hline HGL_RFD ${ }_{\text {rel }}\left(\mathrm{N} / \mathrm{s}^{-1 \mathrm{kgSMM}}\right)$ & 14 & 73.59 & 19.61 & 26.64 & 52.67 & 108.12 & 0.881 & 0.060 \\
\hline HGR_RFD ${ }_{\text {rel }}\left(\mathrm{N} / \mathrm{s}^{-1 \mathrm{kgSMM}}\right)$ & 14 & 74.80 & 20.86 & 27.89 & 53.08 & 125.69 & 0.885 & 0.069 \\
\hline $\mathrm{DL} \_R F D_{\text {rel }}\left(\mathrm{N} / \mathrm{s}^{-1 \mathrm{kgSMM}}\right)$ & 14 & 186.70 & 64.24 & 34.52 & 97.17 & 312.52 & 0.927 & 0.276 \\
\hline $\mathrm{LE}_{-} \mathrm{RFD}_{\text {rel }}\left(\mathrm{N} / \mathrm{s}^{-1 \mathrm{kgSMM}}\right)$ & 14 & 136.52 & 30.65 & 22.45 & 88.07 & 201.22 & 0.967 & 0.831 \\
\hline PF_RFD ${ }_{\text {rel }}\left(\mathrm{N} / \mathrm{s}^{-1 \mathrm{kgSMM}}\right)$ & 14 & 402.60 & 99.41 & 24.69 & 295.66 & 612.96 & 0.847 & 0.020 \\
\hline \multicolumn{9}{|c|}{$\begin{array}{c}\text { Female Judo Athletes } \\
\end{array}$} \\
\hline \multicolumn{9}{|c|}{ Absolute values } \\
\hline & $\mathrm{N}$ & MEAN & SD & $\% \mathrm{cV}$ & MIN & MAX & $\mathrm{S}-\mathrm{W}$ & $\mathrm{p}$ \\
\hline HGL_RFD ${ }_{\max }\left(\mathrm{N} \cdot \mathrm{s}^{-1}\right)$ & 7 & 1651.33 & 717.22 & 43.43 & 953.00 & 2697.00 & 0.876 & 0.209 \\
\hline HGR_RFD ${ }_{\max }\left(\mathrm{N} \cdot \mathrm{s}^{-1}\right)$ & 7 & 1633.00 & 554.26 & 33.94 & 1123.00 & 2650.00 & 0.885 & 0.251 \\
\hline $\mathrm{DL} \_\mathrm{RFD}_{\max }\left(\mathrm{N} \cdot \mathrm{s}^{-1}\right)$ & 7 & 4038.67 & 1446.81 & 35.82 & 2358.00 & 6408.00 & 0.936 & 0.599 \\
\hline LE_RFD ${ }_{\max }\left(\mathrm{N} \cdot \mathrm{s}^{-1}\right)$ & 7 & 3466.00 & 1489.33 & 42.97 & 1999.00 & 6290.00 & 0.896 & 0.308 \\
\hline PF_RFD ${ }_{\max }\left(\mathrm{N} \cdot \mathrm{s}^{-1}\right)$ & 7 & 13026.67 & 5352.60 & 41.09 & 5738.00 & 21376.00 & 0.957 & 0.793 \\
\hline \multicolumn{9}{|c|}{$\begin{array}{r}\text { Relative values } \\
\end{array}$} \\
\hline HGL_RFD ${ }_{\text {rel }}\left(\mathrm{N} / \mathrm{s}^{-1 \mathrm{kgSMM}}\right)$ & 7 & 63.78 & 27.49 & 43.10 & 29.50 & 95.30 & 0.878 & 0.217 \\
\hline HGR_RFD ${ }_{\text {rel }}\left(\mathrm{N} / \mathrm{s}^{-1 \mathrm{kgSMM}}\right)$ & 7 & 62.60 & 20.39 & 32.57 & 39.47 & 93.64 & 0.934 & 0.588 \\
\hline DL_RFD ${ }_{\text {rel }}\left(\mathrm{N} / \mathrm{s}^{-1 \mathrm{kgSMM}}\right)$ & 7 & 152.95 & 47.30 & 30.92 & 87.59 & 226.43 & 0.975 & 0.934 \\
\hline LE_RFD ${ }_{\text {rel }}\left(\mathrm{N} / \mathrm{s}^{-1 \mathrm{kgSMM}}\right)$ & 7 & 133.13 & 53.41 & 40.11 & 61.89 & 222.26 & 0.973 & 0.920 \\
\hline $\begin{array}{l}P F \_R F D_{\text {rel }}\left(\mathrm{N} / \mathrm{s}^{-}\right. \\
\left.{ }_{1 \mathrm{kgSMM}}\right)\end{array}$ & 7 & 495.44 & 189.98 & 38.35 & 221.54 & 768.92 & 0.977 & 0.941 \\
\hline
\end{tabular}


Table 3 shows the initial reference values of the contractile characteristic $F_{\max }$, i.e. maximal isometric muscle force, defined for each of the tested muscle groups in absolute and relative values for both male and female judo athletes.

Table 3 Qualitative standard values for evaluating maximal isometric muscle force $\left(\mathrm{F}_{\max }\right)$

\begin{tabular}{|c|c|c|c|c|c|c|}
\hline \multicolumn{7}{|c|}{ Male Judo Athletes } \\
\hline \multicolumn{7}{|c|}{ Absolute values } \\
\hline MARK & & $\mathrm{HGL}_{\mathrm{N}} \mathrm{F}_{\max }(\mathrm{N})$ & HGR_F $F_{\max }(\mathrm{N})$ & $\mathrm{DL} \mathrm{F}_{\max }(\mathrm{N})$ & LE_F $F_{\max }(\mathrm{N})$ & PF_F $F_{\max }(\mathrm{N})$ \\
\hline Superior & 7 & $>621$ & $>650$ & $>1863$ & $>1660$ & $>4750$ \\
\hline Excellent & 6 & $524-621$ & $551-650$ & $1565-1863$ & $1407-1660$ & $3965-4750$ \\
\hline Good & 5 & $477-524$ & $500-550$ & $1415-1564$ & $1280-1406$ & $3572-3964$ \\
\hline Average & 4 & $380-476$ & $400-499$ & $1116-1414$ & $1025-1279$ & $2785-3571$ \\
\hline Satisfying & 3 & $331-379$ & $349-399$ & $966-1115$ & $898-1024$ & $2392-2784$ \\
\hline Not satisfying & 2 & $233-330$ & $248-348$ & $667-965$ & $643-897$ & $1605-2391$ \\
\hline $\mathrm{Bad}$ & 1 & $<233$ & $<248$ & $<666$ & $<643$ & $<1605$ \\
\hline \multicolumn{7}{|c|}{ Relative values } \\
\hline MARK & & $\begin{array}{c}\mathrm{HGL}_{-} \mathrm{F}_{\mathrm{rel}} \\
\left(\mathrm{N} \cdot \mathrm{kg}_{\mathrm{SMM}^{-1}}{ }^{-1}\right)\end{array}$ & $\begin{array}{c}\text { HGR F }{ }_{\text {rel }} \\
\left(\mathrm{N} \cdot \mathrm{kg}_{\mathrm{SMM}^{-1}}\right)\end{array}$ & $\begin{array}{c}\text { DL_F } \text { rel }_{-1} \\
\left(\mathrm{~N} \cdot \mathrm{kg}_{\mathrm{SMM}}{ }^{-1}\right)\end{array}$ & $\begin{array}{c}\mathrm{LE}_{-} \mathrm{F}_{\mathrm{rel}} \\
\left(\mathrm{N} \cdot \mathrm{kg}_{\mathrm{SMM}}{ }^{-1}\right)\end{array}$ & $\begin{array}{c}\mathrm{PF}_{-} \mathrm{F}_{\text {rel }} \\
\left(\mathrm{N} \cdot \mathrm{kg}_{\mathrm{SMM}}{ }^{-1}\right)\end{array}$ \\
\hline Superior & 7 & $>16.57$ & $>16.78$ & $>48.45$ & $>42.64$ & $>130.97$ \\
\hline Excellent & 6 & $14.30-16.57$ & $14.69-16.78$ & $41.95-48.45$ & $37.45-42.64$ & $110.28-130.97$ \\
\hline Good & 5 & $13.15-14.29$ & $13.64-14.68$ & $38.69-41.94$ & $34.85-37.44$ & $99.94-110.27$ \\
\hline Average & 4 & $10.87-13.14$ & $11.53-13.63$ & $32.17-38.68$ & 29.66-34.84 & 79.24-99.93 \\
\hline Satisfying & 3 & $9.72-10.86$ & $10.48-11.52$ & 28.92-32.16 & $27.06-29.65$ & $68.89-79.23$ \\
\hline Not satisfying & 2 & $7.42-9.71$ & $8.37-10.47$ & 22.39-28.91 & $21.85-27.05$ & $48.19-68.88$ \\
\hline $\mathrm{Bad}$ & 1 & $<7.42$ & $<8.37$ & $<22.39$ & $<21$ & $<48.19$ \\
\hline \multicolumn{7}{|c|}{ Female Judo Athletes } \\
\hline \multicolumn{7}{|c|}{$\begin{array}{c}\text { Absolute values } \\
\end{array}$} \\
\hline MARK & & $\mathrm{HGL}_{\mathrm{N}} \mathrm{F}_{\max }(\mathrm{N})$ & $\mathrm{HGR}_{2} \mathrm{~F}_{\max }(\mathrm{N})$ & $\mathrm{DL} \mathrm{F}_{\max }(\mathrm{N})$ & LE_F $F_{\max }(\mathrm{N})$ & PF_F $F_{\max }(\mathrm{N})$ \\
\hline Superior & 7 & $>370$ & $>337$ & $>867$ & $>833$ & $>4062$ \\
\hline Excellent & 6 & $307-370$ & $290-337$ & $792-867$ & $734-833$ & $3491-4062$ \\
\hline Good & 5 & 274-306 & $267-289$ & $754-791$ & $685-733$ & $3204-3490$ \\
\hline Average & 4 & $209-273$ & $219-266$ & $677-753$ & $585-684$ & $2632-3203$ \\
\hline Satisfying & 3 & $177-208$ & & $639-676$ & $536-584$ & $2346-2631$ \\
\hline Not satisfying & 2 & $111-176$ & $147-194$ & $562-638$ & $435-535$ & $1772-2345$ \\
\hline $\mathrm{Bad}$ & 1 & $<111$ & $<147$ & $<562$ & $<435$ & $<1772$ \\
\hline \multicolumn{7}{|c|}{ Relative values } \\
\hline MARK & & $\begin{array}{c}\mathrm{HGL}_{-} \mathrm{F}_{\mathrm{rel}} \\
\left(\mathrm{N} \cdot \mathrm{kg}_{\mathrm{SMM}}{ }^{-1}\right)\end{array}$ & $\begin{array}{c}\mathrm{HGR}_{-} \mathrm{F}_{\mathrm{rel}} \\
\left(\mathrm{N} \cdot \mathrm{kg}_{\mathrm{SMM}^{-1}}{ }^{-1}\right)\end{array}$ & $\begin{array}{c}\mathrm{DL}_{-} \mathrm{F}_{\mathrm{rel}} \\
\left(\mathrm{N} \cdot \mathrm{kg}_{\mathrm{SMM}}{ }^{-1}\right)\end{array}$ & $\begin{array}{c}\mathrm{LE}_{-} \mathrm{F}_{\mathrm{rel}} \\
\left(\mathrm{N} \cdot \mathrm{kg}_{\mathrm{SMM}}{ }^{-1}\right)\end{array}$ & $\begin{array}{c}\text { PF_F }_{\text {rel }} \\
\left(\mathrm{N} \cdot \mathrm{kg}_{\mathrm{SMM}}{ }^{-1}\right)\end{array}$ \\
\hline Superior & 7 & $>13.89$ & $>13.78$ & $>34.23$ & $>34.11$ & $>153.43$ \\
\hline Excellent & 6 & $11.57-13.89$ & $11.57-13.78$ & $30.83-34.23$ & $29.30-34.11$ & $132.58-153.43$ \\
\hline Good & 5 & $10.40-11.56$ & $10.46-11.56$ & $29.13-30.82$ & 26.89-29.29 & $122.15-132.57$ \\
\hline Average & 4 & $8.07-10.39$ & $8.25-10.45$ & $25.72-29.12$ & $22.07-26.88$ & $101.29-122.14$ \\
\hline Satisfying & 3 & $6.90-8.06$ & $7.14-8.24$ & $24.02-25.71$ & $19.65-22.06$ & $90.85-101.28$ \\
\hline Not satisfying & 2 & $4.56-6.89$ & $4.92-7.13$ & $20.60-24.01$ & $14.82-19.64$ & 69.98-90.84 \\
\hline $\mathrm{Bad}$ & 1 & $<4.56$ & $<4.92$ & $<20.60$ & $<14.82$ & $<69.98$ \\
\hline
\end{tabular}


Table 4 shows the initial reference values of the contractile characteristic $R_{F D} D_{\max }$, i.e. maximal explosive isometric muscle force (explosiveness), defined for each of the tested muscle groups in absolute and relative values for both male and female judo athletes.

Table 4 Qualitative standard values for evaluating maximal isometric explosive muscle force $\left(\mathrm{RFD}_{\max }\right)$

\begin{tabular}{|c|c|c|c|c|c|c|}
\hline \multicolumn{7}{|c|}{ Male Judo Athletes } \\
\hline \multicolumn{7}{|c|}{ Absolute values } \\
\hline MARK & & $\begin{array}{c}\text { HGL_RFD } \\
\left(\mathrm{N} \cdot \mathrm{s}^{-1}\right)\end{array}$ & $\begin{array}{c}\text { HGR_RFD }_{\max } \\
\left(\mathrm{N} \cdot \mathrm{s}^{-1}\right)\end{array}$ & $\begin{array}{c}\text { DL_RFD }_{\max } \\
\left(\mathrm{N} \cdot \mathrm{s}^{-1}\right)\end{array}$ & $\begin{array}{c}\text { LE_RFD }_{\max } \\
\left(\mathrm{N} \cdot \mathrm{s}^{-1}\right)\end{array}$ & $\begin{array}{c}\text { PF_RFD }{ }_{\text {max }} \\
\left(\mathrm{N} \cdot \mathrm{s}^{-1}\right)\end{array}$ \\
\hline Superior & 7 & $>4095$ & $>4250$ & $>12319$ & $>7329$ & $>21520$ \\
\hline Excellent & 6 & $3356-4095$ & $3455-4250$ & $9509-12319$ & $6092-7329$ & $17887-21520$ \\
\hline Good & 5 & $2986-3355$ & $3058-3454$ & $8104-9508$ & $5472-6091$ & $16070-17886$ \\
\hline Average & 4 & $2246-2985$ & $2262-3057$ & $5293-8103$ & $4234-5471$ & 12436-16069 \\
\hline Satisfying & 3 & $1876-2245$ & $1864-2261$ & $3888-5292$ & $3615-4233$ & $10619-12435$ \\
\hline Not satisfying & 2 & $1134-1875$ & $1068-1863$ & $1077-3887$ & $2375-3614$ & 6984-10618 \\
\hline Bad & 1 & $<1134$ & $<1068$ & $<1076$ & $<2375$ & $<6984$ \\
\hline \multicolumn{7}{|c|}{ Relative values } \\
\hline MARK & & $\begin{array}{l}\text { HGL RFD } \\
\left(\mathrm{N} / \mathrm{s}^{-\mathrm{TkgSMM}}\right)\end{array}$ & $\begin{array}{l}\text { HGR RFD } \\
\left(\mathrm{N} / \mathrm{s}^{-\mathrm{IkgSMM}}\right)\end{array}$ & $\begin{array}{l}\text { DL RFD } \\
\left(\mathrm{N} / \mathrm{s}^{-1 \mathrm{kgSMM}}\right)\end{array}$ & $\begin{array}{l}\text { LE RFD } \text { Rel } \\
\left(\mathrm{N} / \mathrm{s}^{-1 \mathrm{kgSMMM}}\right)\end{array}$ & $\begin{array}{l}\text { PF RFD } \\
\left(\mathrm{N} / \mathrm{s}^{-1 \mathrm{kgSMM}}\right)\end{array}$ \\
\hline Superior & 7 & $>112.81$ & $>116.52$ & $>314.54$ & $>197.83$ & $>601.41$ \\
\hline Excellent & 6 & $93.21-112.81$ & $95.67-116.52$ & $250.32-314.54$ & $167.19-197.83$ & $502.02-601.41$ \\
\hline Good & 5 & $83.41-93.20$ & $85.24-95.66$ & $218.20-250.31$ & $151.86-167.18$ & $452.31-502.01$ \\
\hline Average & 4 & $63.80-83.40$ & $64.38-85.23$ & -218.19 & $121.21-151.85$ & $352.91-452.30$ \\
\hline Satisfying & 3 & $53.99-63.79$ & $53.95-64.37$ & $121.84-153.95$ & $105.88-121.20$ & $303.20-352.90$ \\
\hline Not satisfying & 2 & $34.38-53.98$ & $33.08-53.94$ & $57.60-121.83$ & $75.22-105.87$ & 203.78-303.19 \\
\hline Bad & 1 & $<34.38$ & $<33.08$ & $<57.60$ & $<75.22$ & $<203.78$ \\
\hline \multicolumn{7}{|c|}{ Female Judo Athletes } \\
\hline \multicolumn{7}{|c|}{ Absolute values } \\
\hline MARK & & $\begin{array}{c}\text { HGL_RFD }{ }_{\max } \\
\left(\mathrm{N} \cdot \mathrm{s}^{-1}\right)\end{array}$ & $\begin{array}{c}\text { HGR_RFD } \\
\left(\mathrm{N} \cdot \mathrm{s}^{-1}\right)\end{array}$ & $\begin{array}{c}\text { DL_RFD }{ }_{\text {max }} \\
\left(\mathrm{N} \cdot \mathrm{s}^{-1}\right)\end{array}$ & $\begin{array}{c}\mathrm{LE}_{-} \mathrm{RFD}_{\max } \\
\left(\mathrm{N} \cdot \mathrm{s}^{-1}\right)\end{array}$ & $\begin{array}{c}\text { PF_RFD }_{\text {max }} \\
\left(\mathrm{N} \cdot \mathrm{s}^{-1}\right)\end{array}$ \\
\hline Superior & 7 & $>3086$ & $>2142$ & $>6932$ & $>6445$ & $>23732$ \\
\hline Excellent & 6 & $2370-3086$ & $2188-2742$ & $5486-6932$ & $4956-6445$ & $18380-23732$ \\
\hline Good & 5 & $2011-2369$ & $1911-2187$ & -5485 & $4212-4955$ & $15704-18379$ \\
\hline Average & 4 & $1294-2010$ & $1357-1910$ & $3316-4762$ & $2722-4211$ & $10351-15703$ \\
\hline Satisfying & 3 & $935-1293$ & $1080-1356$ & 2593-3315 & $1988-2721$ & 7675-10350 \\
\hline Not satisfying & 2 & 217-934 & $524-1079$ & $1145-2592$ & $487-1977$ & $2321-7674$ \\
\hline Bad & 1 & $<217$ & $<524$ & $<1145$ & $<487$ & $<2321$ \\
\hline \multicolumn{7}{|c|}{ Relative values } \\
\hline MARK & & $\begin{array}{l}\text { HGL RFD } \\
\left(\mathrm{N} / \mathrm{s}^{-\mathrm{lkgSMM}}\right)\end{array}$ & $\begin{array}{l}\text { HGR RFD } \\
\left(\mathrm{N} / \mathrm{s}^{-\mathrm{IkgSMM}}\right)\end{array}$ & $\begin{array}{l}\text { DL RFD } \text { Rel } \\
\left(\mathrm{N} / \mathrm{s}^{-1 / k g S M M}\right)\end{array}$ & $\begin{array}{l}\mathrm{LE} \mathrm{RFD} \text { rel } \\
\left(\mathrm{N} / \mathrm{s}^{-1 \mathrm{kgSMMM}}\right)\end{array}$ & $\begin{array}{l}\text { PFRFD } \\
\left(\mathrm{N} / \mathrm{s}^{-1 \mathrm{kgSMM}}\right)\end{array}$ \\
\hline Superior & 7 & $>118.77$ & $>103.37$ & $>247.54$ & $>239.95$ & $>875.40$ \\
\hline Excellent & 6 & $91.28-118.77$ & 82.99-103.37 & $200.25-247.54$ & $186.55-239.95$ & $685.43-875.40$ \\
\hline Good & 5 & $77.54-91.27$ & $72.80-82.98$ & $176.60-200.24$ & $159.90-186.54$ & 590.44-685.42 \\
\hline Average & 4 & $50.04-77.53$ & $52.42-72.79$ & $129.31-176.59$ & 106.44-159.89 & $400.46-590.43$ \\
\hline Satisfying & 3 & $36.30-50.03$ & $42.22-52.41$ & $105.66-129.30$ & 79.74-106.43 & $305.47-400.45$ \\
\hline Not satisfying & 2 & $8.80-36.29$ & $21.83-42.21$ & $58.35-105.65$ & $26.33-79.73$ & $115.48-305.46$ \\
\hline $\mathrm{Bad}$ & 1 & $<8.80$ & $<21.83$ & $<58.35$ & $<26.32$ & $<115.48$ \\
\hline
\end{tabular}


Table 5 shows the defined model of regression equation of the prediction specification of general fitness from the aspect of maximal isometric muscle force $\left(\mathrm{F}_{\max }\right)$ and relative isometric muscle force $\left(\mathrm{F}_{\text {rel_}} \mathrm{SMM}\right)$ for male $(\mathrm{M})$ and female $(\mathrm{F})$ athletes.

Table 5 The final model for quantitative estimation of maximal isometric muscle force $\left(\mathrm{F}_{\max }\right)$ and relative isometric muscle force $\left(\mathrm{F}_{\text {rel_LSMM }}\right)$ preparedness level for male $(\mathrm{M})$ and female $(\mathrm{F})$ athletes

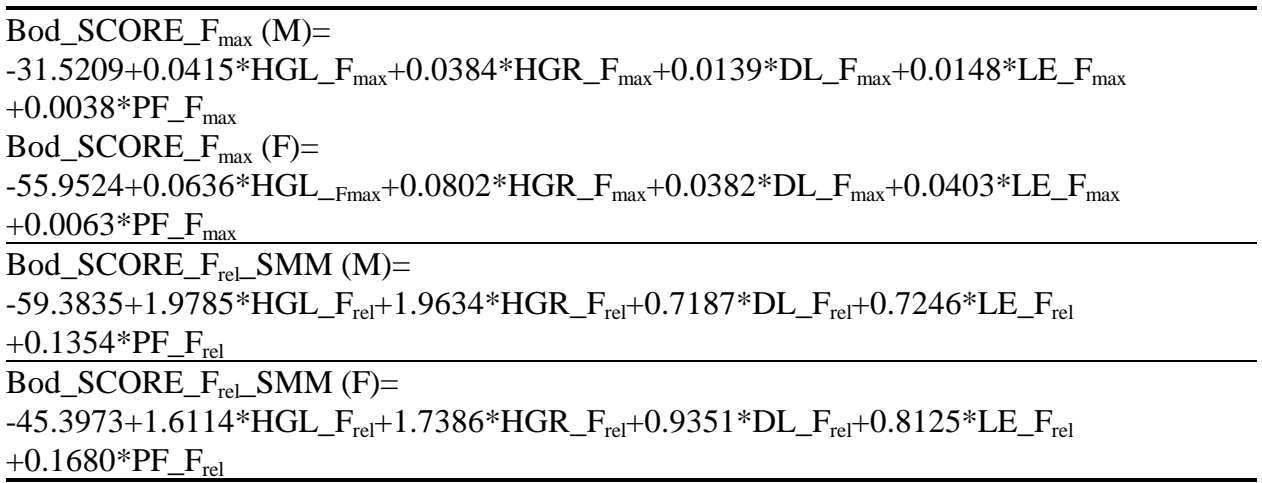

Table 6 shows the defined model of regression equation of the prediction specification of general fitness from the aspect of maximal explosive isometric muscle force $\left(\mathrm{RFD}_{\max }\right)$ and relative explosive isometric muscle force $\left(\mathrm{RFD}_{\max \_} \mathrm{SMM}\right)$ for male $(\mathrm{M})$ and female (F) athletes.

Table 6 The final model for quantitative estimation of maximal explosive isometric muscle force $\left(\mathrm{RFD}_{\max }\right)$ and relative explosive isometric muscle force $\left(\mathrm{RFD}_{\text {max_SMM}}\right.$ SMreparedness level for male $(\mathrm{M})$ and female $(\mathrm{F})$ athletes

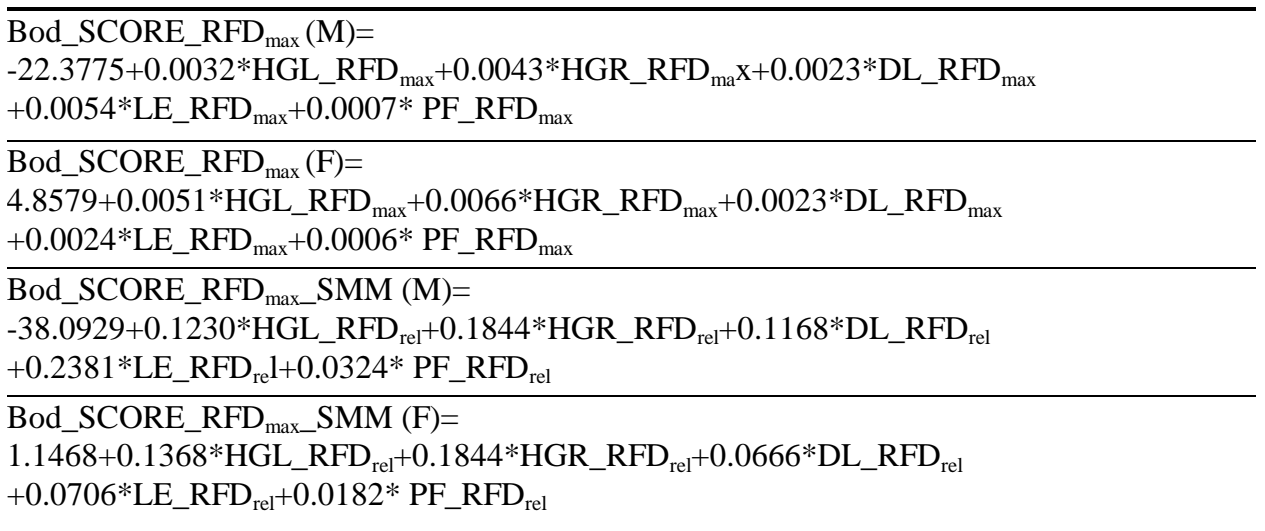




\section{DISCUSSION}

Based on the results of the descriptive statistical analysis it can be concluded that the obtained results are in the acceptable range of variation given the fact that for the contractile characteristic $F_{\max }$, the coefficients of variation range from $16.72 \%$ (HGR_F $\left.F_{\text {rel }}\right)$ to $24.74 \%\left(\right.$ PF_F $\left.F_{\max }\right)$ for the sub-sample of male athletes, while for the sub-sample of female athletes the coefficients of variation for the same contractile characteristic range from $10.64 \%\left(\mathrm{DL}_{-} \mathrm{F}_{\max }\right)$ to $26.86 \%\left(\mathrm{HGL} \mathrm{F}_{\max }\right)$. When observed in relation to contractile characteristic $\mathrm{RFD}_{\max }$, i.e. maximal explosive isometric muscle force, the coefficients of variation of the results range from $22.45 \%\left(\mathrm{LE}_{-} \mathrm{RFD}_{\text {rel }}\right)$ to $41.97 \%\left(\mathrm{DL} \_\mathrm{RFD}_{\max }\right)$ for the male athlete sub-sample, while in the female athlete sub-sample $\% \mathrm{cV}$ ranges from $30.92 \%$ (DL_RFD $D_{\text {rel }}$ ) to $43.43 \%$ (HGL_RFD $\left.{ }_{\max }\right)$. The results of the Shapiro-Wilk test of normality of the distribution have shown a statistically significant deviation of the results in relation to the hypothetical normal curve for the variables $P F \_F_{\text {rel }}(\mathrm{W}=0.768, p=0.002)$ and PF_RFD ${ }_{\text {rel }}(\mathrm{W}=0.847, \mathrm{p}=0.020)$ while for all other variables the distribution of the results was estimated to be approximately normal. On the basis of all aforementioned, it can be argued that the obtained results have average homogeneity (Perić, 2003) and, on a general level, normal distribution, which makes them representative in terms of further scientific interpretation.

When observed in relation to the maximal isometric muscle force of the left hand flexor muscles (HGL_F $\mathrm{F}_{\max }$ ), it can be argued that at the level of the sub-sample of male athletes, average absolute values of the measured results were $427.23 \pm 97.04 \mathrm{~N}$, that is, $12.00 \pm 2.99 \mathrm{~N} \cdot \mathrm{kg}_{\mathrm{SMM}}{ }^{-1}$ relativized in relation to the skeletal muscle mass (SMM). The average result at the level of the female athlete sub-sample for the same muscle group was $240.83 \pm 64.70 \mathrm{~N}$ in relation to the absolute values of the maximal isometric muscle force, that is $9.22 \pm 2.33 \mathrm{~N} \cdot \mathrm{kg}_{\mathrm{SMM}}{ }^{-1}$ relativized in relation to skeletal muscle mass. For the same muscle group, i.e. variable HGL_RFD $\max$, absolute values of maximal explosive muscle force at the level of $2614.85 \pm 740.27 \mathrm{~N} \cdot \mathrm{s}^{-1}$ and $1651.33 \pm 717.22 \mathrm{~N} \cdot \mathrm{s}^{-1}$ were determined for the male and female sub-sample, respectively. Considering relative values of the given contractile characteristic, male athletes achieved an average result of $73.59 \mathrm{~N} / \mathrm{s}^{-1 \mathrm{kgSMM}}$, while female cadet athletes achieved an average result of $63.78 \pm 27.49 \mathrm{~N} / \mathrm{s}^{-1 \mathrm{kgSMM}}$.

In relation to the achieved level of development of the variable HGR_F $F_{\max }$, i.e. maximal isometric muscle force of the right hand flexor muscles, on the basis of the obtained results, it can be argued that the tested male judo athletes have achieved an average result of $448.92 \pm 100.58 \mathrm{~N}$ or $12.57 \pm 2.10 \mathrm{~N} \cdot \mathrm{kg}_{\mathrm{SMM}}{ }^{-1}$ for the relative value of the force in relation to skeletal muscle mass (SMM). Considering the results obtained for the female athlete sub-sample, it can be argued that values determined for the same variable were, on average, at the level of $241.83 \pm 47.61 \mathrm{~N}$ or $9.35 \pm 2.21 \mathrm{~N} \cdot \mathrm{kg}_{\mathrm{SMM}}{ }^{-1}$ relativized in relation to skeletal muscle mass. Considering the contractile characteristic $\mathrm{RFD}_{\max }$, i.e. maximal explosive isometric muscle force, for the variable HGR_RFD ${ }_{\max }$ it can be argued that male athletes have achieved average absolute values of $2658.85 \pm 795.60 \mathrm{~N} \cdot \mathrm{s}^{-1}$ or $74.80 \pm 20.86 \mathrm{~N} / \mathrm{s}^{-1 \mathrm{kgSMM}}$ relativized in relation to skeletal muscle mass. On the level of the female athlete sub-sample it can be concluded that average values of $1633.00 \pm 554.26$ $\mathrm{N} \cdot \mathrm{s}^{-1}$ or $62.60 \pm 20.39 \mathrm{~N} / \mathrm{s}^{-1 \mathrm{kgSMM}}$ were determined.

Regarding the results achieved on the dead lift test, that is, in terms of the achieved level of development of the variable DL_F $F_{\max }$, on the basis of the obtained results it can 
be argued that the tested male judo athletes on average achieve maximum isometric muscle force at the level of 1264.62 $\pm 299.25 \mathrm{~N}$, while female athletes achieved an average value of the given contractile characteristic at the level of $714.50 \pm 76.04 \mathrm{~N}$. Relativized in relation to skeletal muscle mass (SMM), male athletes achieve maximal isometric muscle force at the level of $35.42 \pm 6.52 \mathrm{~N} \cdot \mathrm{kg}_{\mathrm{SMM}}{ }^{-1}$, while females on average achieved $27.41 \pm 3.41 \mathrm{~N} \cdot \mathrm{kg}_{\mathrm{SMM}}{ }^{-1}$. When it comes to the $\mathrm{DL} \_\mathrm{RFD}_{\max }$ variable, that is, maximal explosive isometric muscle force of the same muscle group, average values at the level of $6697.69 \pm 2810.77 \mathrm{~N} \cdot \mathrm{s}^{-1}$ and $4038.67 \pm 1446.81 \mathrm{~N} \cdot \mathrm{s}^{-1}$, were determined for the male and female sub-samples, respectively. Relativized in relation to skeletal muscle mass (SMM), the average values of the obtained results are at the level of $186.70 \pm 64.24 \mathrm{~N} / \mathrm{s}^{-1 \mathrm{kgSMM}}$ and $152.95 \pm 47.30 \mathrm{~N} / \mathrm{s}^{-1 \mathrm{kgSMM}}$ for male and female athletes respectively.

On the leg extension test, male participants on average achieved absolute values of maximal isometric muscle force $\left(\mathrm{LE}_{-} \mathrm{F}_{\max }\right.$ ) on the level of $1151.54 \pm 254.22 \mathrm{~N}$, that is $32.25 \pm 5.20 \mathrm{~N} \cdot \mathrm{kg}_{\mathrm{SMM}}{ }^{-1}$ relative to the mass of skeletal muscles. At the female sub-sample level, for the same muscle group, an average score at the level of $634.00 \pm 99.29 \mathrm{~N}$ was determined, that is, it can be said that the maximal isometric muscle force of the given muscle group relative to the mass of skeletal muscles is on average $24.47 \pm 4.82 \mathrm{~N} \cdot \mathrm{kg}_{\mathrm{SMM}}{ }^{-}$ ${ }^{1}$. For the variable LE_RFD ${ }_{\max }$, that is maximal explosive isometric muscle force of the leg extensor muscles, average values of $4852.23 \pm 1238.52 \mathrm{~N} \cdot \mathrm{s}^{-1}$ and $136.52 \pm 30.65 \mathrm{~N} / \mathrm{s}^{-}$ $1 \mathrm{kgSMM}$, were determined for the male athlete sub-sample considering absolute and values relative to skeletal muscle mass, respectively. For the female athlete sub-sample, average absolute values of the given contractile characteristic were determined at the level of $3466.00 \pm 1489.33 \mathrm{~N} \cdot \mathrm{s}^{-1}$, while in relation to skeletal muscle mass (SMM) the average value of the results was $133.13 \pm 53.41 \mathrm{~N} \cdot \mathrm{s}^{-1}$.

The obtained results for the variable PF_F $F_{\max }$ indicate that at the level of the male athlete sub-sample the average absolute value of the results of the contractile characteristic $F_{\max }$, that is, maximal isometric muscle force of the ankle joint plantar flexor muscles, is at the level of $3177.54 \pm 786.16 \mathrm{~N}$ while for the female athlete subsample the absolute average value at the level of $2917.17 \pm 572.43 \mathrm{~N}$ was determined. When observed relative to the skeletal muscle mass (SMM), male cadets achieved an average score at the level of $89.58 \pm 20.70 \mathrm{~N} \cdot \mathrm{kg}_{\mathrm{SMM}}{ }^{-1}$ while female athletes achieved an average result of $111.71 \pm 20.86 \mathrm{~N} \cdot \mathrm{kg}_{\mathrm{SMM}}{ }^{-1}$. For the variable $\mathrm{PF}_{-} \mathrm{RFD}_{\max }$, that is, maximal explosive isometric muscle force of the ankle joint plantar flexor muscles, average values of $14252.08 \pm 3634.52 \mathrm{~N} \cdot \mathrm{s}^{-1}$ and $402.60 \pm 99.41 \mathrm{~N} / \mathrm{s}^{-1 \mathrm{kgSMM}}$, were determined for the male athlete sub-sample considering absolute and values relative to skeletal muscle mass, respectively. For the female athlete sub-sample average absolute values of the given contractile characteristic were determined at the level of $13026 \pm 5352.60 \mathrm{~N} \cdot \mathrm{s}^{-1}$, while in relation to skeletal muscle mass (SMM), the average value of the results was $495.44 \pm 189.98 \mathrm{~N} \cdot \mathrm{s}^{-1}$.

On the basis of all the afore mentioned, mathematical models were defined for male and female judo athletes' performance considering each of the relevant dimensions of isometric muscle force, i.e. maximal isometric muscle force $\left(\mathrm{F}_{\max }\right)$ and maximal explosive isometric muscle force $\left(\mathrm{RFD}_{\max }\right)$, both in absolute terms and considering results relative to skeletal muscle mass. The defined models have shown absolute predictive potential on a given sample, in all cases explaining $100 \%$ of the measured variance $\left({ }_{\text {Adj }} \mathrm{R}^{2}=1,000\right)$, with minor prediction error values ranging from 0.0000 for Bod_SCORE_F $F_{\max }(\mathrm{M})$ up to 
0.00542 for Bod_SCORE_F rel_SMM $_{\text {S }}$ F). The influence of individual variables on the general point score in each of the defined models, both for maximal isometric muscle force $\left(\mathrm{F}_{\max }\right)$ and maximal explosive isometric muscle force $\left(\mathrm{RFD}_{\max }\right)$, i.e. explosiveness, can be assessed from the values of the Multivariate regression analysis (MRA) coefficients for each of the variables, that is, muscle groups included in the model.

In relation to the previous, a slightly increased influence of the right (HGR) and left (HGL) hand grip variables on a general point score is evident in both male and female maximal isometric muscle force $\left(\mathrm{F}_{\max }\right)$ models. Leg (LE) and back (DL) extensor maximal isometric muscle force relatively equally affects general performance, while plantar flexor muscles (PF) have the least influence (Table 5). For the $\mathrm{RFD}_{\max }$ models, both absolute and relative to skeletal muscle mass, MRA coefficient values indicate that individual muscle groups, i.e. variables, relatively equally affect general performance considering either male or female athletes (Table 6).

\section{CONCLUSION}

The aim of this paper is to define the initial quantitative model and qualitative standard values for evaluation of maximal isometric muscle force $\left(\mathrm{F}_{\max }\right)$ and maximal explosive isometric muscle force $\left(\mathrm{RFD}_{\max }\right)$ in systematically trained and selected cadet judo athletes, both considering absolute values of the obtained results and values derived by relativization of absolute values in relation to skeletal muscle mass (SMM). Given the fact that the coefficient of variation $(\% \mathrm{cV})$, as a relative measure of variability of the data, indicates an average or higher level of homogeneity for all tested variables, and the results of the Shapiro-Wilk test of normality of distribution indicate normal distribution for 38 of the 40 variables it is reasonable to conclude that the obtained results are valid for initial diagnostic use. Taking into account that standard values of the examined contractile characteristics in relation to judo sport and cadet age cannot be found in available scientific literature, and the fact that there is no quantitative data in relation to the methodological aspect of the partialization of results in relation to skeletal muscle mass, the results of this research represent the initial standard values of the measured contractile characteristics, and are primarily aimed to be a basis for qualitative assessment of the level of preparedness of athletes from this aspect. Furthermore, the presented multidimensional models of contractile potential estimation and prediction provide a basis for effective monitoring of training progression and implementation of adequate training routines, all in functions of maximization of athletes' development in this initial stage of sport specialization. In general, all of the afore mentioned will contribute to the increase in the knowledge base in the system of preparation of young judo athletes and possibly lead to consequent improvement of existing technology of sports preparation in judo sport.

Acknowledgment: The paper is a part of the project "Effects of the Applied Physical Activity on Locomotor, Metabolic, Psychosocial and Educational Status of the Population of the Republic of Serbia", number III47015, funded by the Ministry of Education, Science and Technological Development of the Republic of Serbia - Scientific Projects 2011 - 2018 Cycle. 


\section{REFERENCES}

Blais, L., Trilles, F., \& Lacouture, P. (2007). Validation of a specific machine to the strength training of judokas. Journal of Strength and Conditioning Research, 21(2), 409-412.

Calmet, M., Miarka, B., \& Franchini, E. (2010). Modeling of grasps in judo contests. International Journal of Performance Analysis in Sport, 10(3), 229-240.

Cuk, Š., Bratić, M., \& Mučibabić, M. (2016). The motor potential of young judokas in Slovenia. Facta Universitatis, series: Physical Education and Sport, 14 (2), 297-305.

Detanico, D., Budal Arins, F., Dal Pupo, J., \& Dos Santos, S.G. (2012). Strength parameters in judo athletes: an approach using hand dominance and weight categories. Human Movement, 13(4), 330-336.

Dopsaj, M., Milošević, M., Blagojević, M. (2000). Analysis of the reliability and factoral validity of selected muscle force mechanical characteristics during isometric multi joint test. In Y. Hong, \& D.P. Johns (Eds.), Proceedings of XVIII International Simposium on Biomechanics in Sport, (146-149). Hong Kong: Department of sports science and physical education - The Chinese University of Hong Kong.

Dopsaj, M., Koropanovski, N., Vučković, G., Blagojević, M., Marinković, B., \& Miljuš, D. (2007). Maximal isometric hand grip force in well-trained university students in Serbia: Descriptive, functional and sexual dimorphic model. Serbian Journal of Sports Sciences, 1(4), 138-147.

Dopsaj, M., Ćopić, N., Nešić, G., \& Sikimić, M. (2010). A simple mathematical model for estimating general jumping preparedness of senior female volleyball players. Exercise and Quality of Life, 2(2), 63-74.

Dopsaj, M. (2015). Analitika i dijagnostika u sportu i fizičkom vaspitanju (Analytics and diagnostics in sports and physical education). Faculty of Sport and Physical Education, University of Belgrade. In Serbian

Dopsaj, M., Ilić, V., Đorđević-Nikić, M., Vučković, M., Eminović, F., Macura, M., \& Ilić, D. (2015). Descriptive model and gender dimorphism of body structure of physically active students of Belgrade University: Pilot study. Anthropologist, 19(1), 239-248.

Franchini, E., Nunes, A. V., Moraes, J. M., \& Del Vecchio, F. B. (2007). Physical fitness and anthropometrical profile of the Brazilian male judo team. Journal of Physiological Anthropology, 26(2), 59-67.

Franchini, E., Del Vecchio, F.B., Matsushigue, K.A., \& Artioli, G.G. (2011). Physiological profiles of elite judo athletes. Sports Medicine, 41(2), 147-166.

Ivanović, J., \& Dopsaj, M. (2013). Reliability of force-time curve characteristics during maximal isometric leg press in differently trained high-level athletes. Measurement, 46(7), 2146-2154.

Koprivica, V. (2013). Teorija sportskog treninga (Theory of sports training). Autonomous Edition of Author: Belgrade. In Serbian

Laskowski, R., \& Suchanowski, A. (2010). Maximal strength development in a yearly training cycle of judo competitors. Journal of Combat Sports and Martial Arts, 1(2), 41-44.

Marcon, G., Franchini, E., Jardim, J.R., \& Barros Neto, T.L. (2010). Structural analysis of action and time in sports: judo. Journal of Quantitative Analysis in Sports, 6(4).

Marković, S., Dopsaj, M., Jovanović, S. (2017). Multidimensional model of general contractile potential estimation of basic muscle groups in cadet judo athletes. In S. Pantelić (Ed.). International Congress "FIS Comunications", Book of Procedings (pp. 55-61). Niš: Faculty of Sport and Physical Education, University of Niš.

Milišić, B. (2003). Upravljanje treningom (Training management). SIP: Belgrade. In Serbian

Monteiro, L., Chambel, L., \& Cardoso, M. (2011). Elite and sub-elite judokas: the factors behind international success. In A.A. Figueiredo \& C. Gutierrez-Garcia (Ed.). Scientific congress on martial arts and combat sports, proceedings (pp.73-76). Viseau: Instituto Politecnico de Viseau.

Monteiro, L. (2016). Analysis of rate force development, power and resistance explosive strength indicators in top elite vs. elite male judokas. In H. Sertić, S. Črak \& Segedi (Eds.). Applicable Research in Judo Proceedings book, pp. 36-39. Poreč: Faculty of Kinesiology, University of Zagreb.

Nurkić, M., Bratić, M., Mitić, D., Kafentarakis, I. (2017). The differences in the motor area of cadet and junior age judokas. Facta Universitatis, series: Physical Education and Sport, 15 (1), 83-92.

Perić, D. (2003). Statistika primenjena u sportu i fizičkom vaspitanju (Statistics applied in sports and physical education). Belgrade: Faculty of Sport and Physical Education. In Serbian

Pocecco, E., Ruedl, G., Stankovic, N., Sterkowicz, S., Del Vecchio, F.B., Gutiérrez-García, C., \& Menz, V. (2013). Injuries in judo: a systematic literature review including suggestions for prevention. British Journal of Sports Medicine, 47(18), 1139-1143.

Saraiva, A.R., Reis, V.M., Costa, P.B., Bentes, C.M., Costa e Silva, G.V., \& Novaes, J.S. (2014). Chronic effects of different resistance training exercise orders on flexibility in elite judo athletes. Journal of Human Kinetics, 40(1), 129-137. 
Sindik, J., Rendulić, D., Čorak, S., \& Lewis A.P. (2014). Analysis of the relevant factors of retaining women in judo. Montenegrin Journal of Sports Science and Medicine, 3(2), 23-31

Sport and organization rules of the International Judo Federation (Edition 2017). (2017). Laussane, Switzerland: International Judo Federation.

Stamenković, S., Stanković, N., Nurkić, M., Nikolić, D., \& Petković, E. (2016). The difference in some motor skills between judokas and non-athletes of an early school age. Facta Universitatis, Series Physical Education and Sport, 14 (2), 201-209.

Thomas, S.G., Cox, M.H., LeGal, Y.M., Verde, T.J., \& Smith, H.K. (1989). Physiological profiles of the Canadian National Judo Team. Canadian Journal of Sport Sciences, 14(3), 142-147.

Zatsiorsky, V. (1982). Спортивная метрлогия (Sports metrology). Moscow: Physical Education and Sports. In Russian

\section{KVALITATIVNA I KVANTITATIVNA PROCENA KARAKTERISTIKA IZOMETRIJSKE MIŠIĆNE SILE RAZLIČITIH MIŠIĆNIH GRUPA KOD DŽUDISTA KADETSKOG UZRASTA: MULTIDIMENZIONALNI MODEL U ODNOSU NA POL}

Cilj ovog rada je da se definiše incijalni kvantitativni i kvalitativni model za procenu bazičnih karakteristika izometrijske mišićne sile (jačine) kod sistematski treniranih i selektiranih džudista kadetskog uzrasta. U ovom istraživanju razmatrane su apsolutne vrednosti dobijenih rezultata kao $i$ izvedene vrednosti dobijene relativizacijom rezultata u odnsou na masu skeletnih mišića (SMM). Osnovni metod primenjen u ovom istraživanju je bilo laboratorijsko testiranje. Svi podaci su prikupljeni primenom izometrijske tenziometrije, pomoću tenziometrijskih sondi. Uzorak ispitanika u ovom radu činilo je 21 sportista kadetskog uzrasta, od čega 14 muškaraca i 7 žena. Sva merenja su izvršena primenom standardizovanih procedura testiranja na sledećim mišićnim grupama: mišići pregibači prstiju leve (HGL) i desne (HGR) šake, mišići opružači leđa (DL) i nogu (LE) i mišići plantarni fleksori skočnog zgloba (PF). Na osnovu dobijenih rezultata definisani su odvojeni multidimenzionalni matematički modeli za procenu nivoa pripremljenosti i kontraktilnog potencijala za obe osnovne karakteristike izometrijske mišićne sile: maksimalnu izometrijsku mišićnu silu $\left(F_{\max }\right) i$ maksimalnu ekplozivnu izometrijsku mišićnu silu (RFD $\left.{ }_{\max }\right)$. Kvalitativna procena kontraktilnog potencijala za svaku od testiranih mišićnih grupa, odnosno varijabli, je omogućena definisanjem normativnih vrednosti za 7 nivoa pripremljenosti za obe osnovne kontraktilne karakteristike mišićne sile, kako za muške, tako i za ženske takmičare kadetskog uzrasta u džudo sportu.

Ključne reči: džudo, kadeti, izometrijska mišićna sila, normativne vrednosti, model 